\title{
Should clinical cardiologists incorporate the proximal isovelocity surface area (PISA) method into their ever enlarging armamentarium?
}

This issue contains a paper comparing measurement of ventricular septal defect shunt ratio by a proximal isovelocity surface area technique (usually abbreviated as PISA) method and by the Fick method. ${ }^{1}$ The authors used some shortcuts in the PISA method, perhaps to make the measurements easier and more appealing to the cardiologist, and demonstrated a reasonable correlation of shunt calculation by the two techniques.

\section{Measurement of flows}

Although the PISA technique and theory may be only minimally familiar to many cardiologists, non-invasive measurement of flow, forward or regurgitant, has long been a goal of physicians treating patients with cardiac malformations. Various techniques for flow computation have been reported and all have limitations. For the echocardiographer, the earliest methods used assumptions about ventricular shape. ${ }^{2}$ Such assumptions worked well for normal ventricles and somewhat less well for diseased or excessive volume loaded ventricles. Doppler computations provided the next major wave of enthusiasm for non-invasive measurement of cardiac output, ${ }^{34}$ but these methods required accurate measurements of flow area and velocity. The product of flow area $\left(\mathrm{cm}^{2}\right)$ and velocity $(\mathrm{cm} / \mathrm{s})$ is flow $\left(\mathrm{cm}^{3} / \mathrm{s}\right)$ : Although both flow area and velocity can be measured in many instances, examples in which one or both cannot be measured, particularly in hearts with malformations, limited the usefulness of this technique. Furthermore, measurement of shunting or regurgitation require determination of the difference between two flows, thus increasing the opportunity for error. An excellent example of a difficult measurement is determining the shunt through a septal defect.

Echocardiographic imaging of the size of the defect does not provide an estimate of the shunt volume that passes through the defect. Further, echocardiographic sizing of atrial defects for device closure has demonstrated the inadequacy of this method. ${ }^{5}$ Qualitative estimation of shunt size by colour Doppler may also be misleading. Accordingly, such two dimensional echocardiographic measurements of defect size and shunt magnitude provide a mere approximation of the actual situation.

\section{Proximal isovelocity surface area}

AN INTRODUCTION

The search for the Holy Grail of accurate flow measurements led, at the beginning of the 1990s, to detailed investigation of the PISA technique. ${ }^{67}$ It has been long known that velocities accelerate and converge in radial streamlines before an obstruction. If the rate of isovelocities can be measured and if PISA can be determined accurately, application of the familiar flow formula (flow area $x$ velocity) can be applied. Further, the continuity equation assures, absent loss of fluid, that the volume flow rate on either side of an orifice is identical. Clearly, the isovelocity lines can be measured by assessing the velocity of aliasing proximal to an obstruction and the radius of aliasing can be assessed by lowering the velocity range of interrogation as shown in fig 1 of the paper by Kurotobi et al. ${ }^{1}$

\section{CHARACTERISTICS OF PROXIMAL ISOVELOCITY SURFACE} AREA

Is the application of PISA deceptively too simple? All methods have positive features and drawbacks. Even PISA is not immune to the laws of physics. (1) Assumptions are based on passage of non-viscous fluid through a very small orifice. (2) The shape of the PISA is inconstant at a range of flow rates. Critical flow rate for forming a PISA is determined by the product of orifice size and aliasing velocity. Below a critical flow rate (indexed by orifice size) PISA cannot be detected, and at critical flow rate PISA is flat. These shape characteristics usually prevent use of PISA in non-restricted flow, but make it applicable to restrictive orifices in which higher flow rates produce a hemispheric flow closer to the orifice (but still $>1$ orifice diameter proximal to the orifice) and a more hemielliptical PISA still further from the orifice. Zhang et $a l^{8}$ also demonstrated that the first alias proximal to the orifice, in a more hemispheriodal field, overestimated flow rate, and the third alias, in the more hemielliptic field, underestimated flow rate, but the second velocity alias provided more accurate volume flow information. (3) The radius measurement is for maximal velocity and a volume flow event that occurs over the whole of systole would require integration of temporal velocities over systole rather than just a peak measurement. Temporal integration is usually not done, but the authors in the present paper took systolic duration into account. (4) Measurement of the alias radius is not easy, even when employing zoom. (5) Cape $e t$ $a l^{9}$ demonstrated that recorded velocity with respect to the transducer is a combination of orifice movement and velocity relating to flow convergence. This combination, depending upon the direction of orifice movement, could add or subtract from the true convergence aliasing velocity.

What is the future of proximal isovelocity surface area?

The PISA method in the past has been used mainly in investigational protocols for assessing the peak flow rate of mitral regurgitation and, early in the experience, for assessing ventricular septal defect peak flow rate. ${ }^{10} \mathrm{~W}$ ill it be adopted by the cardiology community for general clinical use? The most attractive features are that it may be computed relatively rapidly during the course of a clinical echocardiogram and that it may provide a reasonably reli- 
able measurement, particularly for serial follow up of a given patient. However, the field of cardiology is littered with remnants of seldom or never employed techniques for approximating a clinically important data point. Only time will tell whether PISA will be adopted by the clinical community, relegated to investigational protocols, or forgotten. However, the technique clearly has promise and demonstrated usefulness.

Pediatric Cardiology,

University of Arizona,

Tucson, Arizona, USA

1 Kurotobi S, Sano T, Matsushita T, Takeuchi M, Kogaki S, Miwatani T, et al. Quantitative, non-invasive assessment of ventricular septal defect shunt flow by measuring proximal isovelocity surface area on colour Doppler mapping. Heart $1997 ; 78: 305-9$.

2 Pombo JF, Troy BL, Russel RO. Left ventricular volumes and ejection fraction by echocardiography. Circulation 1971;43:480-90.

3 Goldberg SJ, Sahn DJ, Allen HD, Valdes-Cruz LM, Hoenecke H, Carnahan Y. Evaluation of pulmonary and systemic blood flow by twodimensional Doppler echocardiography using fast Fourier transform spectral analysis. Am $\mathcal{F}$ Cardiol 1982;50:1394-1400.

4 Huntsman LL, Stewart DK, Barnes SR, Franklin SB, Colocousis JS, Hessel EA. Noninvasive Doppler determination of cardiac output in man: clinical validation. Circulation 1983;67:593-602.

5 Reddy SC, Rao PS, Ewenko J, Koscik R, Wilson AD. Echocardiographic predictors of success of catheter closure of atrial septal defect with the buttoned device. Am Heart f 1995;129:76-82.

6 Okamoto $M$, Tsubokura T, Nakagawa $H$, Morichika N, Amioka $H$, Yamagata $T$, et al The suction signal detected by Doppler echocardiography in patients with mitral regurgitation: its clinical significance. f Cardiol 1988;18:739-46.

7 Utsunomiya T, Ogawa T, Tang HA, Doshi R, Patel D, Ouan M, et al. Doppler color flow mapping of the proximal isovelocity surface area: a new method for measuring volume flow rate across a narrowed orifice. new method for measuring volume

8 Zhang J, Jones M, Shandes R, Valdes-Cruz LM, Murillo A, Yamada I, et al. Accuracy of flow convergence estimates of mitral regurgitation obtained by use of multiple color flow Doppler M-mode aliasing boundobtained by use of multiple color flow Doppler M-mode aliasing

9 Cape EG, Kim YH, Heinrich RS, Grimes RY, Muralidharan E, Broder JD, et al. Cardiac motion can alter proximal isovelocity surface area calculaet al. Cardiac motion can alter proximal isovelocity surface

10 Moises VA, Maciel BC, Hornberger LK, Murillo-Olives A, Valdes-Cruz $\mathrm{LM}$, Sahn DJ, et al. A new method for noninvasive estimation of ventricular septal defect shunt flow by Doppler color flow mapping: imaging of the laminar flow convergence region on the left septal surface. $\mathcal{F} \mathrm{Am} \mathrm{Coll}$ Cardiol 1991;18:824-32. 